Supplementary material for

\title{
Solvent Extraction of Superfine Pulverized Coal. Part 2.
}

\section{Free Radical Characteristics}

\author{
Jiaxun Liu ${ }^{\dagger}$, Xinyu Zhong ${ }^{\dagger}$, Xiumin Jiang ${ }^{\dagger}$, Xue Jiang ${ }^{\ddagger}, * *$ \\ ${ }^{\dagger}$ School of Mechanical Engineering, Shanghai Jiao Tong University, Shanghai 200240, China \\ $¥$ School of Life Sciences, East China Normal University, Shanghai 200241, China
}

\section{Six Tables}

Table S1 The free radical concentration of the extract

Table S2 The free radical concentration of the raw coal and residues

Table S3 The $g$ value of the extract

Table S4 The $g$ value of the raw coal and residues

Table S5 The linewidth of the extract

Table S6 The linewidth of the raw coal and residues

\footnotetext{
${ }^{*}$ Corresponding authors. Tel: +86 2154344867.

E-mail address: xjiang@bio.ecnu.edu.cn (X. Jiang).
}

Notes: The authors declare no competing financial interest. 
Table S1. The free radical concentration of the extract.

\begin{tabular}{ccccccccc}
\hline & \multicolumn{3}{c}{ pyl (spin/g) } & & & \multicolumn{2}{c}{ thfl (spin/g) } \\
\hline & Lorenzian1 & Lorenzian2 & Lorenzian3 & Gaussian & Lorenzian1 & Lorenzian2 & Lorenzian3 & Gaussian \\
\hline HN_8.1 & $3.86614 \mathrm{E} 17$ & $5.2327 \mathrm{E} 17$ & $3.74969 \mathrm{E} 17$ & $2.29289 \mathrm{E} 18$ & $4.92509 \mathrm{E} 17$ & $6.82993 \mathrm{E} 17$ & $4.85149 \mathrm{E} 17$ & $2.85347 \mathrm{E} 18$ \\
HN_14.1 & $2.86634 \mathrm{E} 17$ & $3.71769 \mathrm{E} 17$ & $2.63242 \mathrm{E} 17$ & $1.6148 \mathrm{E} 18$ & $2.36978 \mathrm{E} 17$ & $3.19693 \mathrm{E} 17$ & $2.3939 \mathrm{E} 17$ & $1.21237 \mathrm{E} 18$ \\
HN_23.9 & $2.45219 \mathrm{E} 17$ & $3.26537 \mathrm{E} 17$ & $2.40657 \mathrm{E} 17$ & $1.40106 \mathrm{E} 18$ & $1.40589 \mathrm{E} 17$ & $1.68171 \mathrm{E} 17$ & $1.17053 \mathrm{E} 17$ & $8.04845 \mathrm{E} 17$ \\
HN_34.7 & $1.77425 \mathrm{E} 17$ & $2.66688 \mathrm{E} 17$ & $2.20688 \mathrm{E} 17$ & $9.80004 \mathrm{E} 17$ & $2.19113 \mathrm{E} 17$ & $2.48554 \mathrm{E} 17$ & $4.42922 \mathrm{E} 16$ & $8.82013 \mathrm{E} 17$ \\
NMG_11 & $5.3212 \mathrm{E} 17$ & $7.68942 \mathrm{E} 17$ & $5.89949 \mathrm{E} 17$ & $3.82933 \mathrm{E} 18$ & $1.72407 \mathrm{E} 17$ & $1.95864 \mathrm{E} 17$ & $5.06045 \mathrm{E} 16$ & $1.47899 \mathrm{E} 18$ \\
NMG_13.6 & $1.35942 \mathrm{E} 17$ & $2.00706 \mathrm{E} 17$ & $1.58615 \mathrm{E} 17$ & $1.07534 \mathrm{E} 18$ & $1.55826 \mathrm{E} 17$ & $1.76229 \mathrm{E} 17$ & $3.72613 \mathrm{E} 16$ & $1.44122 \mathrm{E} 18$ \\
NMG_18.7 & $2.97099 \mathrm{E} 16$ & $2.72732 \mathrm{E} 16$ & $2.76291 \mathrm{E} 15$ & $1.98946 \mathrm{E} 17$ & $1.70512 \mathrm{E} 17$ & $1.9953 \mathrm{E} 17$ & $7.55011 \mathrm{E} 16$ & $1.88771 \mathrm{E} 18$ \\
NMG_21.6 & $1.35332 \mathrm{E} 17$ & $1.40338 \mathrm{E} 17$ & $1.39402 \mathrm{E} 16$ & $9.09614 \mathrm{E} 17$ & $1.71657 \mathrm{E} 17$ & $1.90468 \mathrm{E} 17$ & $5.49221 \mathrm{E} 16$ & $1.66623 \mathrm{E} 18$ \\
\hline
\end{tabular}


Table S2. The free radical concentration of the raw coal and residues.

\begin{tabular}{cccccccccccccc}
\hline \multicolumn{9}{c}{ rc (spin/g) } & \multicolumn{4}{c}{ pys (spin/g) } & \multicolumn{1}{c}{ thfs (spin/g) } \\
\hline & Lorenzian1 & Lorenzian2 & Lorenzian3 & Gaussian & Lorenzian1 & Lorenzian2 & Lorenzian3 & Gaussian & Lorenzian1 & Lorenzian2 & Lorenzian3 & Gaussian \\
\hline HN_8.1 & $8.72746 \mathrm{E} 18$ & $1.08543 \mathrm{E} 19$ & $5.74835 \mathrm{E} 18$ & $1.30502 \mathrm{E} 19$ & $6.01658 \mathrm{E} 18$ & $6.47479 \mathrm{E} 18$ & $3.15535 \mathrm{E} 18$ & $8.52236 \mathrm{E} 18$ & $1.10775 \mathrm{E} 19$ & $1.30242 \mathrm{E} 19$ & $6.52029 \mathrm{E} 18$ & $1.61496 \mathrm{E} 19$ \\
HN_14.1 & $8.84001 \mathrm{E} 18$ & $1.16343 \mathrm{E} 19$ & $6.2241 \mathrm{E} 18$ & $1.40752 \mathrm{E} 19$ & $1.00258 \mathrm{E} 19$ & $1.29612 \mathrm{E} 19$ & $6.58912 \mathrm{E} 18$ & $1.67431 \mathrm{E} 19$ & $6.88447 \mathrm{E} 18$ & $8.43848 \mathrm{E} 18$ & $4.21487 \mathrm{E} 18$ & $1.12561 \mathrm{E} 19$ \\
HN_23.9 & $5.19605 \mathrm{E} 18$ & $6.7173 \mathrm{E} 18$ & $3.53118 \mathrm{E} 18$ & $8.29322 \mathrm{E} 18$ & $6.31659 \mathrm{E} 18$ & $7.95233 \mathrm{E} 18$ & $3.96521 \mathrm{E} 18$ & $1.04069 \mathrm{E} 19$ & $5.6252 \mathrm{E} 18$ & $7.5116 \mathrm{E} 18$ & $3.94654 \mathrm{E} 18$ & $8.33484 \mathrm{E} 18$ \\
HN_34.7 & $7.34546 \mathrm{E} 18$ & $9.66401 \mathrm{E} 18$ & $5.16375 \mathrm{E} 18$ & $1.19752 \mathrm{E} 19$ & $1.04784 \mathrm{E} 19$ & $1.3537 \mathrm{E} 19$ & $6.70579 \mathrm{E} 18$ & $1.64036 \mathrm{E} 19$ & $6.92555 \mathrm{E} 18$ & $9.47557 \mathrm{E} 18$ & $5.00729 \mathrm{E} 18$ & $1.07732 \mathrm{E} 19$ \\
NMG_11 & $7.63027 \mathrm{E} 18$ & $6.38657 \mathrm{E} 18$ & $3.14496 \mathrm{E} 18$ & $1.08824 \mathrm{E} 19$ & $4.38505 \mathrm{E} 18$ & $3.33162 \mathrm{E} 18$ & $1.51828 \mathrm{E} 18$ & $7.13088 \mathrm{E} 18$ & $4.95413 \mathrm{E} 18$ & $4.14531 \mathrm{E} 18$ & $1.96798 \mathrm{E} 18$ & $7.25026 \mathrm{E} 18$ \\
NMG_13.6 & $7.59885 \mathrm{E} 18$ & $6.84938 \mathrm{E} 18$ & $3.4326 \mathrm{E} 18$ & $1.28851 \mathrm{E} 19$ & $4.32897 \mathrm{E} 18$ & $3.44451 \mathrm{E} 18$ & $1.57598 \mathrm{E} 18$ & $7.75771 \mathrm{E} 18$ & $5.15327 \mathrm{E} 18$ & $3.87086 \mathrm{E} 18$ & $1.71682 \mathrm{E} 18$ & $9.78298 \mathrm{E} 18$ \\
NMG_18.7 & $8.23123 \mathrm{E} 18$ & $7.81438 \mathrm{E} 18$ & $3.90987 \mathrm{E} 18$ & $1.34667 \mathrm{E} 19$ & $4.42327 \mathrm{E} 18$ & $3.47696 \mathrm{E} 18$ & $1.57494 \mathrm{E} 18$ & $7.58062 \mathrm{E} 18$ & $4.35794 \mathrm{E} 18$ & $3.3376 \mathrm{E} 18$ & $1.46414 \mathrm{E} 18$ & $8.43962 \mathrm{E} 18$ \\
NMG_21.6 & $5.52222 \mathrm{E} 18$ & $4.46285 \mathrm{E} 18$ & $2.12998 \mathrm{E} 18$ & $8.50219 \mathrm{E} 18$ & $3.24053 \mathrm{E} 18$ & $2.74953 \mathrm{E} 18$ & $1.3387 \mathrm{E} 18$ & $5.1212 \mathrm{E} 18$ & $3.06728 \mathrm{E} 18$ & $2.36357 \mathrm{E} 18$ & $1.03193 \mathrm{E} 18$ & $6.25611 \mathrm{E} 18$ \\
\hline
\end{tabular}


Table S3. The $g$ value of the extract.

\begin{tabular}{ccccccccc}
\hline \multicolumn{7}{c}{ pyl } & \multicolumn{3}{c}{ thfl } \\
\hline & Lorenzian1 & Lorenzian2 & Lorenzian3 & Gaussian & Lorenzian1 & Lorenzian2 & Lorenzian3 & Gaussian \\
\hline HN_8.1 & 2.00552 & 2.00458 & 2.00368 & 2.00451 & 2.00523 & 2.00427 & 2.00336 & 2.00421 \\
HN_14.1 & 2.00563 & 2.00465 & 2.00371 & 2.00464 & 2.00564 & 2.00466 & 2.00371 & 2.00461 \\
HN_23.9 & 2.0057 & 2.00472 & 2.00378 & 2.00467 & 2.00526 & 2.00431 & 2.00338 & 2.00439 \\
HN_34.7 & 2.00582 & 2.00484 & 2.00384 & 2.00475 & 2.00608 & 2.00508 & 2.00405 & 2.00467 \\
NMG_11 & 2.00556 & 2.00462 & 2.00372 & 2.00447 & 2.00552 & 2.00461 & 2.00365 & 2.00414 \\
NMG_13.6 & 2.00537 & 2.00447 & 2.00354 & 2.00429 & 2.00582 & 2.0049 & 2.00397 & 2.00451 \\
NMG_18.7 & 2.00566 & 2.00476 & 2.00386 & 2.00457 & 2.00559 & 2.00467 & 2.00372 & 2.00416 \\
NMG_21.6 & 2.00571 & 2.00482 & 2.00391 & 2.0045 & 2.00583 & 2.00491 & 2.00397 & 2.00445 \\
\hline
\end{tabular}


Table S4. The $g$ value of the raw coal and residues.

\begin{tabular}{|c|c|c|c|c|c|c|c|c|c|c|c|c|}
\hline & \multicolumn{4}{|c|}{ rc } & \multicolumn{4}{|c|}{ pys } & \multicolumn{4}{|c|}{ thfs } \\
\hline & Lorenzian1 & Lorenzian2 & Lorenzian3 & Gaussian & Lorenzian 1 & Lorenzian2 & Lorenzian3 & Gaussian & Lorenzian1 & Lorenzian2 & Lorenzian3 & Gaussiar \\
\hline HN_8.1 & 2.0054 & 2.00417 & 2.0029 & 2.00449 & 2.00531 & 2.00418 & 2.00303 & 2.00452 & 2.00532 & 2.00416 & 2.00297 & 2.00447 \\
\hline HN_14.1 & 2.00546 & 2.00419 & 2.0029 & 2.00451 & 2.00541 & 2.00425 & 2.00307 & 2.00456 & 2.0053 & 2.00414 & 2.00293 & 2.00449 \\
\hline HN_23.9 & 2.00546 & 2.00421 & 2.00294 & 2.00458 & 2.00543 & 2.00428 & 2.00308 & 2.00461 & 2.00548 & 2.00416 & 2.00283 & 2.00457 \\
\hline HN_34.7 & 2.00556 & 2.00428 & 2.00301 & 2.00458 & 2.00559 & 2.00442 & 2.00324 & 2.00474 & 2.00546 & 2.0042 & 2.00289 & 2.00451 \\
\hline NMG_11 & 2.00553 & 2.00428 & 2.00302 & 2.00455 & 2.00528 & 2.00423 & 2.00322 & 2.00448 & 2.0055 & 2.00431 & 2.00309 & 2.00456 \\
\hline NMG_13.6 & 2.00553 & 2.00429 & 2.00305 & 2.00452 & 2.00531 & 2.00426 & 2.00325 & 2.00448 & 2.00529 & 2.00421 & 2.00314 & 2.00449 \\
\hline NMG_18.7 & 2.00558 & 2.0043 & 2.00302 & 2.00453 & 2.00529 & 2.00425 & 2.00321 & 2.0045 & 2.00548 & 2.00416 & 2.00283 & 2.00457 \\
\hline NMG_21.6 & 2.00551 & 2.00424 & 2.00297 & 2.00462 & 2.00551 & 2.00437 & 2.00326 & 2.00464 & 2.00528 & 2.0042 & 2.0031 & 2.00449 \\
\hline
\end{tabular}


Table S5. The linewidth of the extract.

\begin{tabular}{ccccccccc}
\hline \multicolumn{9}{c}{ pyl (G) } \\
\hline & Lorenzian1 & Lorenzian2 & Lorenzian3 & Gaussian & Lorenzian1 & Lorenzian2 & Lorenzian3 & Gaussian \\
\hline HN_8.1 & 3.11555 & 2.89295 & 3.04694 & 8.47461 & 3.1658 & 2.94011 & 3.10281 & 8.5603 \\
HN_14.1 & 3.24561 & 2.98403 & 3.09638 & 8.71029 & 3.27669 & 3.06657 & 3.25337 & 8.76823 \\
HN_23.9 & 3.26391 & 3.0247 & 3.17189 & 8.73057 & 3.17431 & 2.86923 & 2.9627 & 8.65069 \\
HN_34.7 & 3.28046 & 3.15727 & 3.43878 & 8.74863 & 3.98224 & 4.02713 & 6.08022 & 8.26534 \\
NMG_11 & 2.98389 & 2.83539 & 3.03479 & 8.32491 & 3.2374 & 3.46283 & 4.34425 & 7.68778 \\
NMG_13.6 & 2.83813 & 2.79748 & 3.05065 & 7.92937 & 3.1248 & 3.3382 & 3.58763 & 7.58016 \\
NMG_18.7 & 3.33649 & 3.2847 & 2.60772 & 7.59261 & 3.00377 & 3.24901 & 4.35522 & 7.52859 \\
NMG_21.6 & 3.32147 & 3.39786 & 3.38297 & 7.58962 & 3.10725 & 3.31076 & 4.12592 & 7.60369 \\
\hline
\end{tabular}


Table S6. The linewidth of the raw coal and residues.

\begin{tabular}{|c|c|c|c|c|c|c|c|c|c|c|c|c|}
\hline & \multicolumn{4}{|c|}{$\operatorname{rc}(G)$} & \multicolumn{4}{|c|}{ pys (G) } & \multicolumn{4}{|c|}{ thfs $(G)$} \\
\hline & Lorenzian1 & Lorenzian2 & Lorenzian3 & Gaussian & Lorenzian1 & Lorenzian2 & Lorenzian3 & Gaussian & Lorenzian1 & Lorenzian2 & Lorenzian3 & Gaussian \\
\hline HN_8.1 & 5.33363 & 4.50991 & 4.85039 & 13.17248 & 5.01988 & 4.09101 & 4.30752 & 11.74545 & 5.10868 & 4.24412 & 4.50594 & 12.18252 \\
\hline HN_14.1 & 5.37666 & 4.59659 & 4.98609 & 13.78493 & 4.98039 & 4.21777 & 4.54039 & 12.49199 & 4.9765 & 4.17766 & 4.46502 & 12.4739 \\
\hline HN_23.9 & 5.30097 & 4.51913 & 4.8962 & 13.67512 & 4.97731 & 4.18649 & 4.48886 & 12.48406 & 5.62179 & 4.80244 & 5.24431 & 14.5888 \\
\hline HN_34.7 & 5.40359 & 4.61367 & 5.00705 & 13.96584 & 5.11254 & 4.30624 & 4.63429 & 12.72933 & 5.45629 & 4.6763 & 5.11192 & 14.1292 \\
\hline NMG_11 & 5.63739 & 4.42443 & 4.46986 & 11.43662 & 4.73115 & 3.52882 & 3.4885 & 9.2634 & 5.40334 & 4.21559 & 4.25651 & 11.04332 \\
\hline NMG_13.6 & 5.38185 & 4.30381 & 4.36908 & 11.33203 & 4.64361 & 3.49945 & 3.47036 & 9.22708 & 4.83724 & 3.60708 & 3.5601 & 9.61968 \\
\hline NMG_18.7 & 5.55786 & 4.49733 & 4.59897 & 11.7573 & 4.71932 & 3.55341 & 3.5279 & 9.39085 & 4.89427 & 3.64836 & 3.60872 & 9.71166 \\
\hline NMG_21.6 & 5.5556 & 4.31131 & 4.33546 & 11.58522 & 4.958 & 3.85308 & 3.88706 & 10.40449 & 4.83064 & 3.61068 & 3.56291 & 9.71894 \\
\hline
\end{tabular}


